\title{
INHALTLICHE WAHLPROGRAMMANALYSE DER PARTEIEN HINSICHTLICH DER INTEGRATIONSTHEMEN ZUR BUNDESTAGSWAHL 2013 IN DEUTSCHLAND
}

\author{
Dr. Phil. Mustafa ACAR \\ Forschungszentrum für Türkei-EU-Deutschland (Zf-TEUD) \\ (Türkiye-Avrupa Birliği-Almanya Araştırmaları Merkezi)
}

\begin{abstract}
ABSTRAKT
Das Ziel der Untersuchung besteht darin, die Wahlprogramme der Parteien zur Bundestagswahl 2013 in Deutschland hinsichtlich der Integrationspolitik, des Rechtsextremismus, der Diskriminierung und der EU-Beitrittsfrage der Türkei quantitativ und qualitativ inhaltlich $z u$ analysieren. Diese Themen werden von den Türken als die wichtigen Schwerpunkte der Wahl angesehen. Während die Integrationspolitik der CDU und CSU konservativ ist, haben die SPD, Grünen, Linkspartei und FDP eine progressive Integrationspolitik. Doppelstaatsbürgerschaft und kommunales Wahlrecht von Ausländern werden von allen Parteien außer der CDUCSU akzeptiert. Obwohl die SPD, Grünen und FDP die Türkei für eine EUVollmitgliedschaft unterstützen, wird diese Möglichkeit von den Unionsparteien abgelehnt. Die Linkspartei vermeidet eine Aussage zu dieser Frage. Alle Parteien außer der CDU-CSU betonen den Kampf gegen Rechtsextremismus und Diskriminierung. Diese Probleme werden von der SPD, Grünen und Linkspartei besonders detailliert thematisiert, dagegen werden von den Unionsparteien nicht, von der FDP wenig bearbeitet. Die Wahlprogramme der linken Parteien sind hinsichtlich der ausgewählten Themen viel versprechend.

Schlüsselwörter: Wahlprogrammen, Parteien, Inhaltsanalyse, Bundestagswahl, Integrationspolitik, Diskriminierung, Rechtsextremismus.
\end{abstract}

\section{ALMANYA GENEL SEÇIMLERINDE PARTILERIN SEÇIM PROGRAMLARININ UYUM KONULARI BAKIMINDAN İÇERİK ANALIZİ}

ÖZ

Alman partilerinin 2013 genel seçimlerine yönelik seçim programlarının, uyum politikaları, aşırı să̆, ayrımcılık ve Türkiye'nin AB üyeliği konularında nicel ve nitel olarak içerik analizi bu araştırmanın amacını oluşturmaktadır. Bu konular, Almanya'da yaşayan Türkler tarafindan çok önemli görülmekte ve seçmen davranışlarını partilerin bu konulardaki tutumlar belirlemektedir. Uyum politikaları konusunda muhafazakar bir tutum takınan Hıristiyan Demokratlar (CDU-CSU), çifte vatandaşlı̆̆l, yerel seçimlerde yabancıların oy hakkını ve Türkiye'nin AB üyeliğini reddederken, aşırı să̆ terör ve ayrımcılık konularına hiç değinmemektedir Buna karşın Sosyal Demokratların 
(SPD), Yeşillerin, Sol Partinin ve Liberallerin (FDP) daha özgürlükcü ve liberal bir uyum politikası formüle ettikleri görülmektedir. Çifte vatandaşlı ve yerel seçimlerde yabancıların oy hakkı SPD, Yeşiller ve Liberaller tarafindan kabul edilirken, bu partiler Türkiye'nin AB üyeliğine de destek vermektedirler. Sol Parti bütün seçimlerde oy hakkını ve çok vatandaşlı̆̆ talep ederken, Sosyal Demokratlar ve Yeşillerle birlikte aşırı sağa ve ayrımcılı̆̆a karşı mücadeleyi en uzun ve detaylı işleyen parti olarak dikkat çekiyor.

Anahtar Kelimeler: Seçim Programı, Partiler, İçerik Analizi, Almanya Genel Seçimleri, Uyum Politikası, Ayrımcılık, Aşırı Să̆.

\section{EINLEITUNG}

Vor den Wahlen auf den Bundes-, Landes-, und Kommunalebenen veröffentlichen die Parteien ein Wahlprogramm, das die Politikangebote der Parteien für die Wähler ist. Die Wahlprogramme der Parteien können als ,das Themenmanagement des Programmwahlkampfes“ (Korte, 2003: 122) bezeichnet werden und sind im Allgemeinen „handlungsorientiert, zeitlich begrenzt und relativ umfassend“ (Klingemann, 1989: 99). In diesem Sinne ist „ein Wahlprogramm ein Dokument, das über Charakter und Ziele einer politischen Gruppe, Partei oder Bewegung Aufschluss geben soll“" (Stammen, 1986: 17). Während die Programmatik ,ein konstitutives Merkmal politischer Parteien ist und eine Partei durch ihre Programmatik ihre politische Identität gewinnt und sichert“ (Klingemann, 1989: 99), informieren sich die Wähler durch die Wahlprogramme über die Zielsetzungen und Positionen in den Politikfeldern der unterschiedlichen Parteien. In diesem Zusammenhang beinhalten die Wahlprogramme die Zielerklärungen über die Schwerpunktthemen, die im Falle eines Wahlsieges politisch realisiert werden. Im wesentlich erfüllt das Wahlprogramm zwei Funktionen: „Die Programme der Parteien haben einerseits die Aufgabe, nach außen zu wirken und andererseits die Funktion, das innere Gefüge der Parteien zu gestalten“ (Stammen, 1986: 17). Die Entstehung der Wahlprogramme ist ein Prozess innerhalb der Parteien. Die Entwürfe der Wahlprogramme der Parteien werden von Programmkommissionen oder Fachausschüssen in den Parteien erarbeitet. An den Vorbereitungsprozessen beteiligen die regionalen Untergliederungen der Parteien, die verschiedenen Arbeitsgemeinschaften und Vorfeldorganisationen. Am Ende des Prozesses werden die Programme durch die Parteitagsdelegierten beschlossen. Für den Beschluss der Wahlprogramme ist die Einstimmigkeit ein wichtiger Faktor, "um die Einheit und Geschlossenheit der Partei zu demonstrieren" (Klingemann und Volkens, 2001: 513)

Die Wahlprogramme der Parteien, als der Gegenstand der wissenschaftlichen Forschung, wurden ab 1949 von den Wissenschaftlern durch die qualitativen und quantitativen Methoden analysiert. Die Untersuchungen von Klingemann (1989), von Klingemann und Volkens (2001), von Volkens (1989, 2002), von Pappi und Shikano (2004) und Debus (2007) thematisieren 
die wahlprogrammatischen Positionen der Parteien auf den unterschiedlichen politischen Ebenen, die Zeitspezifischen Gemeinsamkeiten der Wahlprogramme und die ideologischen Unterschiede der Parteien auf einer Links-RechtsDimension. Diese Inhaltsanalysen sind besonders hilfreich, um ideologische Grundpositionen der Parteien im Politikfeld Migration zu verstehen. Seit „mit der NPD in den Jahren 1967-68 die politische Diskussion über Ausländer aufkam" (Vogel und Wüst, 2003: 266), beschäftigen sich die Parteien immer mehr mit der Migrations- und der Integrationspolitik und politisieren dieses Thema. In diesen Diskussionen nimmt die türkische Bevölkerung eine besondere Stellung ein, weil sie die Mehrzahl der Ausländer in Deutschland bildet und die allgemeine Politik „bislang nur unzureichend auf die besondere Problemlage dieser Personen reagiert" (Wilamowitz-Moellendorff, 2005: 40). Obwohl in den 1980er Jahren ,die Ausländerfragen vor allem bei Landtagswahlkämpfen wiederholt eine wichtige Rolle spielte“ (Vogel und Wüst, 2003: 267), wurde dieses Thema erst mit den 1990er Jahren in die Bundestagswahlen eingebracht.

Die Zielsetzung dieser Untersuchung ist die Wahlprogramme der Parteien zur Bundestagswahl 2013 hinsichtlich der Integrationspolitik, des Rechtsextremismus, der Diskriminierung und der EU-Beitrittsfrage der Türkei, quantitativ und qualitativ inhaltlich zu analysieren. Diese Themen werden von den Parteien in ihren Wahlprogrammen mehr oder weniger immer thematisiert, weil die türkischstämmige Wählerschaft bei den Bundestagswahlen von diesen Themen besonders mobilisiert wird. Wenn es sich bei der Wählerschaft um eine mit Migrationshintergrund handelt, schließt sich unmittelbar die Frage an, welche politischen Probleme oder Themen ihr besonders wichtig sind. Es sind vor allem drei Themen, die das Wahlverhalten der türkischstämmigen Wähler beeinflussen: „Verhinderung von Diskriminierung, der EU-Beitritt der Türkei und der Kampf gegen Rechtsradikalismus" (Wilamowitz-Moellendorff, 2005: 43). Diese politischen Probleme werden „von $63,4 \%$ der Männer und von $72,3 \%$ der Frauen sehr wichtig gefunden“" (Acar, 2012: 65). In diesem Zusammenhang stehen die Fragen im Zentrum dieser Untersuchung:Von welchen Parteien werden die ausgewählten Themen in Wahlprogrammen behandelt und in welchem Umfang? Welche Positionsunterschiede gibt es zwischen den Parteien in dieser Hinsicht? Wie weit sind die Parteien programmatisch voneinander entfernt?

In dieser Untersuchung wird im ersten Schritt die Methode der quantitativen und qualitativen Inhaltsanalyse erläutert, mit der Wahlprogramme wissenschaftlich untersucht werden. Danach werden die Wahlprogramme der Parteien unter den Kategorien Integrationspolitik, Rechtsextremismus, Diskriminierung und EU-Beitrittsfrage der Türkei analysiert und vergleicht, um die gegebenen Fragen zu antworten. Die Analyse schließt mit dem Resümee. 


\section{METHODE UND FORSCHUNGSDESIGN}

Empirische Sozialforschung wird als "die systematische Erfassung und Deutung sozialer Erscheinungen" bezeichnet und "unter Methoden der empirischen Sozialforschung" versteht man "die geregelte nachvollziehbare Anwendung von Erfassungsinstrumenten wie Befragung, Beobachtung und Inhaltsanalyse" (Atteslander, 2003: 5). In diesem Zusammenhang ist die Inhaltsanalyse ein sozialwissenschaftliches Forschungsinstrument. Die Definition der Inhaltsanalyse besteht die Schwierigkeiten, weil die unterschiedlichen Ansätze die Inhaltsanalyse nach ihren Schwerpunkten definieren. Im Allgemeinen ist "die Inhaltsanalyse eine Forschungstechnik für die objektive, systematische und quantitative Beschreibung des manifesten Inhalts von Kommunikation" (Berelson, 1952: 18). Diese Definition wird von Mayring (1990: 11-12) mit sechs Spezifika folgendermaßen detailliert:

"- Die Inhaltsanalyse hat Kommunikation zum Gegenstand.

- Die Inhaltsanalyse arbeitet mit Texten, Bildern, Noten- also mit symbolischem Material. Gegenstand der Analyse ist somit eine fixierte Kommunikation.

- Das systematische Vorgehen ist von wesentlicher Bedeutung.

- Die Systematik zeigt sich in der Regelgeleitetheit. Die Analyse läuft nach expliziten Regeln ab.

- Eine gute Inhaltsanalyse geht theoriegeleitet vor. Sowohl die Ergebnisse als auch die einzelnen Analyseschritte sind von theoretischen Überlegungen geleitet.

- Inhaltsanalyse will durch Aussagen über das zu analysierende Material Rückschlüsse auf bestimmte Aspekte der Kommunikation ziehen.”

Es handelt sich bei der Inhaltsanalyse um qualitative und quantitative Methoden. Während die quantitative Inhaltsanalyse "auf einer Interpretation sozialer Sachverhalte beruht, die in der Beschreibung der Sachverhalte durch Zahlen resultiert", befasst sich die qualitative Inhaltsanalyse mit der „Interpretation sozialer Sachverhalte, die in einer verbalen Beschreibung dieser Sachverhalte resultiert" (Gläser und Laudel, 2006: 24). Die quantitative Inhaltsanalyse soll „objektiv, systematisch, quantitativ und manifest“ (Lamnek, 2005: 494) sein. Mit der qualitativen Inhaltsanalyse werden „Texte systematisch analysiert, indem sie das Material schrittweise mit theoriegeleiteten am Material entwickelten Kategoriensystemen bearbeitet" (Mayring, 2002: 114).

Für die Qualität und Wissenschaftlichkeit der Forschung wurden einige Kriterien entwickelt, die als „Zielvorgaben und Prüfsteine“ (Lamnek, 2005: 142) einer Methode bezeichnet werden. Obwohl diese Kriterien mehrheitlich für die quantitative Forschung gültig sind, können diese Gütekriterien auf die qualitative Forschung übertragen werden (vgl. Mayring, 1990: 100f; Flick, 1991: 4; Kromrey, 1998:514). Diese Kriterien können sich in vier Kategorien gliedern: 
- Objektivität: Die Unabhängigkeit der Ergebnisse vom Forscher und die Erforderung der Transparenz (vgl. Bortz und Döring, 2005: 194).

- Generalisierbarkeit: Die Übertragbarkeit der theoretischen Erkenntnisse auf praktische Kontexte (vgl. Steinke, 1999: 246).

- Validität: Das Maß für die Brauchbarkeit von Forschungsmethoden (vgl. Atteslander 2010: 228).

- Zuverlässigkeit: Ein Maß für die Reproduzierbarkeit von Messergebnissen (vgl. Diekmann, 2001: 217).

Obwohl die quantitative Inhaltsanalyse stark mit Zahlen, Daten und Messwerten beschäftigt ist, versucht man mit der qualitativen Inhaltsanalyse als Auswertungsverfahren, das zu untersuchende Material detailliert, ganzheitlich, erfassend und interpretativ $\mathrm{zu}$ bewerten. Im Zentrum der qualitativen Inhaltsanalyse steht die interpretative Ausdeutung der Analyse und ein Kategoriensystem in einem Wechselverhältnis zwischen der Fragestellung und dem zu forschenden Material. Nach Mayring (2002: 115) gibt es drei unterschiedliche inhaltsanalytische Verfahren.

- Zusammenfassende Inhaltsanalyse: Durch die zusammenfassende Inhaltsanalyse wird „das Material reduziert, dass die wesentlichen Inhalte erhalten bleiben und durch Abstraktion ein überschaubares Corpus zu schaffen, das immer noch ein Abbild des Grundmaterials ist" (Mayring, 1988: 53).

- Explikative Inhaltsanalyse: Nach Mayring (2002: 115) ist die explikative Inhaltsanalyse ,zu einzelnen fraglichen Textteilen (Begriffen, Sätzen, ) zusätzliches Material heranzutragen, das das Verständnis erweitert, das die Textstelle erläutert, erklärt, ausdeutet." Es handelt sich bei der explikativen Inhaltsanalyse um die enge und weitere Inhaltsanalyse.

- Strukturierende Inhaltsanalyse: Es ist hier das Ziel, „eine bestimmte Struktur aus dem Material herauszufiltern. Das können formale Aspekte, inhaltliche Aspekte oder bestimmte Typen sein; es kann aber auch eine Skalierung, eine Einschätzung auf bestimmten Dimensionen, angestrebt werden“ (Mayring 2002: 118):

Für diese Untersuchung wird die quantitative und qualitative Inhaltsanalyse als die Untersuchungsmethode ausgewählt. "Allerdings ist es nahezu unmöglich beide Verfahren strikt zu trennen, da meistens eine Mischform benötigt wird, so dass es lediglich darauf ankommt, welche Form der Kategorienbildung stärker gewichtet wird“ (Früh, 2007: 73). Die quantitative Inhaltsanalyse dient hier zur Antwort dieser Forschungsfrage: In welchem Umfang behandeln die Parteien die ausgewählten Themen in ihren Wahlprogrammen? Die explikative Inhaltsanalyse im engeren und weiteren Sinne hilft der Analyse der wahlprogrammatischen Positionsunterschiede der Parteien. Während man unter dem engeren Textkontext „das direkte Textumfeld der interpretationsbedürftigen Textstelle“ versteht, "können der weitere Textkontext als die über den Text hinausgehenden Informationen über Textverfasser, Adressaten, Interpreten, kulturelles Umfeld; auch nonverbales 
Material und die Informationen über Entstehungssituation eingehen“ (Mayring, 2002: 117f.). Man versteht hier die explikative Inhaltsanalyse auch als Kontextanalyse der fraglichen Textteile.

In diesem Zusammenhang läuft die Analyse folgendermaßen ab:

- Die Bestimmung des Materials: Die Wahlprogramme der CDU-CSU, SPD, FDP, LINKE und GRÜNEN zur Bundestagswahl 2013.

- Die Beschreibung des Materials: Titel, Seitenzahl, Veröffentlichungsart usw.

- Die quantitative Inhaltsanalyse: Die Zahlenwerte der ausgewählten Themen (der Integrationspolitik, des Rechtsextremismus, der Diskriminierung und der EU-Beitrittsfrage der Türkei) innerhalb des gesamten Materials.

- Die Kategorien: Die Parteien lassen sich nach der Stimmenzahl bei der letzten Bundestagswahl (2009) einordnen. Unter den eingeordneten Parteien werden die Wahlprogramme in vier Kategorien qualitativ analysiert: Integrationspolitik, Rechtsextremismus, Diskriminierung und EU-Beitrittsfrage der Türkei.

- Die Überprüfung der Explikation: Es wird hier überprüft, ob die Explikation ausreichend ist. Wenn die Explikation nicht ausreichend ist, werden die weiteren Explikationsmateriale zugelassen, um die Untersuchungsfragen zu beantworten.

\section{INHALTSANALYSE}

\section{A. Allgemeine Beschreibung der Wahlprogramme}

Die Wahlprogramme aller Parteien, die meistens auch türkisch zusammengefasst werden, implizieren die migrations- und integrationsspezifischen Themen. Aber diese Themen werden in den Wahlprogrammen der Parteien oberflächlich behandelt und bilden nicht den Kern der Programme.

Tabelle 1: Allgemeine Beschreibung der Wahlprogramme

\begin{tabular}{|l|l|l|l|l|l|}
\hline & $\begin{array}{c}\text { CDU- } \\
\text { CSU }\end{array}$ & SPD & FDP & LINKE & GRÜNE \\
\hline Seitenzahl & 128 & 120 & 94 & 86 & 337 \\
\hline Veröffentlichungsdatum & 23.06 & $\begin{array}{l}14.04 . \\
2013\end{array}$ & $\begin{array}{l}27.06 . \\
2013\end{array}$ & $\begin{array}{l}16.06 . \\
2013\end{array}$ & $\begin{array}{l}28.04 . \\
2013\end{array}$ \\
\hline Veröffentlichungsart & PDF & PDF & PDF & PDF & PDF \\
\hline Gesamtwörterzahl & 42.299 & 43.633 & 38.628 & 30.655 & 90.258 \\
\hline
\end{tabular}

Die Wahlprogramme der Parteien wurden neben den normalen Druck auch auf ihren Internetseiten als PDF-Datei veröffentlicht. Für diese Untersuchung werden diese PDF-Dateien verwendet. Die Sprache der Programme ist klar und verständlich. SPD und CDU benennen ihre Programme als das Regierungsprogramm. 
Die Programme sind im Einzelnen (vgl. Tabelle 1):

- Am 23.06.2013 haben CDU (Christlich Demokratische Union) und CSU (Christlich-Soziale Union) ihr gemeinsames Wahlprogramm unter dem Titel „Gemeinsam erfolgreich für Deutschland. Regierungsprogramm 20132017“" verabschiedet. Das Programm ist 128 Seiten, besteht aus 42.299 Wörtern und sieben Abschnitten. Für dieses Programm hat die CDU den Bürgern eine Möglichkeit geboten, um ihre Wünsche und Ideen zum Wahlprogramm einzubringen. Darum wurde eine Internetseite unter dem Titel „Was mir am Herzen liegt" aufgestellt.

- Der Titel des Wahlprogramms der SPD (Sozialdemokratische Partei Deutschlands) lautet „Das wir entscheidet. Das Regierungsprogramm 20132017“،. Dieses Programm wurde am 14.04.2013 auf dem Bundesparteitag der SPD einstimmig akzeptiert und veröffentlicht. Dieses Programm beinhaltet 120 Seite, 43.633 Wörter und 11 Abschnitte. Die SPD hat erstmals die Menschen aufgerufen, das Wahlprogramm mitzugestalten. Auf bundesweit mehr als 350 Veranstaltungen hat die SPD gefragt: Was muss in Deutschland besser werden? Die Vorschläge und Ideen wurden von der SPD im Programm berücksichtigt.

- Das Wahlprogramm der FDP (Die Freien Demokraten) wurde auf dem Bundesparteitag vom 4. bis 5. Mai 2013 in Nürnberg beschlossen und am 27.06.2013 unter dem Titel „, Bürgerprogramm 2013. Damit Deutschland stark bleibt. Nur mit uns." online veröffentlicht. Im 94-seitigen Programm sind sieben Abschnitte und 38.628 Wörter enthalten. Die FDP hat auch die Menschen aufgerufen, sich an der Diskussion um das Wahlprogramm zu beteiligen.

- Die Linkspartei hat am 16.06.2013 auf dem Parteitag in Dresden ihr Wahlprogramm unter dem Titel „100\% Sozial“" mit großer Mehrheit verabschiedet. Die Menschen wurden im März mit den regionalen Konferenzen über die Inhalt des Wahlprogramms informiert. Mit der Seitenzahl (86), Gesamtwörterzahl (30.655) und den Abschnitten (6) hat die Linkspartei ein kürzeres Wahlprogramm als andere Parteien.

- Das Wahlprogramm vom Bündnis 90/Die Grünen wurde am 28.04.2013 auf der Bundesdelegiertenkonferenz in Berlin beschlossen. Der Titel des Programms lautet „Zeit für den grünen Wandel. Teilhaben. Einmischen. Zukunft Schaffen“. Im Vergleich zu anderen Parteien haben die Grünen das längste Programm. Das 337-seitige Programm besteht aus 90.258 Wörtern und 19 Abschnitten. Im März haben die Grünen einen Entwurf des Programms veröffentlicht und dieser Entwurf wurde von der Öffentlichkeit und den Parteimitgliedern diskutiert.

\section{B. Integrationspolitik}

Im Wahlprogramm von CDU und CSU werden die Bezeichnungen „Zuwanderer“, „Menschen mit Zuwanderergeschichte“ und „Ausländer“ verwendet., während die SPD im Allgemeinen von „Menschen“ spricht. Die FDP bezeichnet die Ausländer als „Menschen mit Migrationshintergrund“ und 
„Mitbürger“. Die Grünen und die Linkspartei verwenden in ihren Programmen die Ausdrücke „Migrantinnen und Migranten“.

Tabelle 2: Integrationspolitik der deutschen Parteien

\begin{tabular}{|l|l|l|}
\hline \multicolumn{1}{r|}{ Parteien } & \multicolumn{2}{|c|}{ Integrationspolitik } \\
\hline & \% & Wörter \\
\hline CDU-CSU & 2,50 & $(1058)$ \\
\hline SPD & 2,43 & $(1061)$ \\
\hline FDP & 3,39 & $(1310)$ \\
\hline DIE LINKE & 1,31 & $(404)$ \\
\hline DIE GRÜNEN & 0,59 & $(533)$ \\
\hline
\end{tabular}

Während die CDU und CSU die Zulassung der doppelten Staatsbürgerschaft ablehnen, akzeptieren die SPD, die Grünen, die Linkspartei und die FDP die doppelte Staatsbürgerschaft. Nach SPD und FDP ist die Hinnahme der doppelten Staatsbürgerschaft nur möglich, wenn die Menschen mindestens seit 5 Jahren in Deutschland leben. Obwohl ein kommunales Wahlrecht für Ausländer von der Unionsparteien abgelehnt wird, setzen sich SPD, FDP und Grüne für ein kommunales Wahlrecht für Nicht-EUBürgerInnen ein. Die Linkspartei will Wahlrecht für in Deutschland lebende Migrantinnen und Migranten auf Bundes-, Landes- und kommunaler Ebene.

Obwohl die CDU und CSU die Integrationspolitik mit 2,50\% noch länger als die anderen Parteien außerhalb der FDP thematisieren, haben sie eine konservative Integrationspolitik und betonen mehrheitlich die Aufgaben anstatt der Rechte von Migranten. Deutschland wird als „ein erfolgreiches Integrationsland" bezeichnet. Sie orientieren ihre Integrationspolitik an den gemeinsamen deutschen Werten und der Kultur, die eine schnelle und erfolgreiche Integration ermöglicht. Sie wollen weitere hochqualifizierte und leistungsbereite Menschen aus anderen Ländern für sich gewinnen. Man braucht ausreichende Informationsangebote etwa zu Einreise, Schule und Beruf sowie zum Spracherwerb, damit sich Neuzuwanderer auf das Leben in Deutschland gut vorbereiten können. Sie werben dafür, dass sich mehr junge Menschen mit Zuwanderungsgeschichte für eine berufliche Laufbahn im öffentlichen Dienst, insbesondere als Lehrerinnen und Lehrer, als Polizistinnen und Polizisten oder in der Justiz entscheiden. Die CDU und CSU betonen gute Deutschkenntnisse als der Schlüssel für eine erfolgreiche Integration und fördern frühes Erlernen der deutschen Sprache. Deutsche Sprache wird von den Unionsparteien als die Stärkung der Identität und des Zusammenhalts in der deutschen Gesellschaft angesehen. Niemand hat ohne deutsche Sprache Zukunftschancen in Deutschland. Die Einbürgerung ist das Bekenntnis zu Deutschland und dessen 
Werten. Die Annahme deutscher Staatsangehörigkeit ist ein starkes Zeichen der Zugehörigkeit, die die vollen staatsbürgerlichen Rechte und Pflichten bringt und stärkster Ausdruck einer erfolgreichen Integration ist. Die Unionsparteien betonen, dass sie der Abschottung in Parallelgesellschaften und islamischen Sondergerichten und den Integrationsverweigerern entgegentreten.

Deutschland ist laut der SPD sowohl ein offenes Land, als auch ein Einwanderungsland und muss den nächsten Schritt tun und ein Einbürgerungsland werden. Sie setzt sich für ein gleichberechtigtes und gesellschaftliches Miteinander in Vielfalt ein. Integrationspolitik heißt für die SPD, neu zu denken, den Begriff der Integration zu überwinden und durch den selbstverständlichen gesellschaftspolitischen Anspruch auf Teilhabe und Partizipation zu setzen. Diesem Prinzip müssen sich alle Ressorts der Bundesregierung verpflichten. Deutschland braucht sowohl eine Willkommenskultur als auch eine Teilhabestruktur und nur so können Freiheit, Chancengleichheit, Bildung und Arbeit für alle geschaffen werden. Die SPD tritt dafür ein, dass das erfolgreiche Miteinander vor allem von sozialen Faktoren abhängt, dass Unterschiede in der Herkunft, Religion oder Kultur nicht verdrängt werden dürfen, dass eine offene Gesellschaft im Rahmen der Grundrechte allen Religionen den Freiraum zur Entfaltung ihres Glaubens bietet und dass rechtliche Gleichstellung und Chancengerechtigkeit die Grundlagen einer offenen Gesellschaft sind. Das Ziel der SPD ist es, dass die kulturelle, weltanschauliche und religiöse Vielfalt innerhalb der rechtsstaatlichen und sozialstaatlichen Grundordnung in Politik und Verwaltung, Wirtschaft, Kunst und Kultur selbstverständlich mitgedacht wird. Insbesondere will die SPD, dass Deutschlands Kinder auch deutsche Staatsbürger sind und bleiben. Mädchen und Jungen, die hier geboren werden, sollen die deutsche Staatsangehörigkeit erhalten und behalten. Es wird ermöglicht, ausländischen Studierenden ohne Einschränkungen in Deutschland $\mathrm{zu}$ arbeiten. Die Verbesserung der Integrationskurse, die Erleichterung des Familiennachzuges und die Öffnung des öffentlichen Dienstes für die Menschen mit Migrationshintergrund werden im Regierungsprogramm der SPD betont.

Die FDP behandelt die Integrationspolitik in ihrem Wahlprogramm mit $3,39 \%$ am längsten. Die Liberalen akzeptieren, dass Deutschland ein Einwanderungsland und Zuhause für die Migranten ist. Die Herkunftsländer von Menschen sind unwichtig. Deutschland muss Hindernisse abbauen und Türen öffnen. Zur Teilhabe für das Miteinander in der deutschen Gesellschaft muss deutsche Sprache, die der Schlüssel zur Bildung, zum Erfolg und zu gesellschaftlicher Integration ist, gelernt werden und demokratische Grundordnung von Deutschland akzeptiert werden. Toleranz, Offenheit und eine Kultur des Miteinanders werden von der FDP als liberale Werte angesehen und sehr oft betont. Aber es gibt die Grenzen der Toleranz. Z.b. Zwangsheirat ist verbot. Zur Integration sollen die Mittel für die Integrationskurse, Kinderbetreuung, sprachliche und berufliche Bildung erhöht werden. 
Integration ist unmöglich, ohne gemeinsame Sprache und die Akzeptanz der republikanischen Werte der Verfassung. Deutschland soll für Talente aus aller Welt offen sein. Die FDP will das Entfallen der Arbeitserlaubnispflicht, den Aufenthaltstitel für die ausländischen Studierenden, eine Möglichkeit zur beschleunigten Einbürgerung nach vier Jahren und die Stärkung der Familien. Die Liberalen fordern den offenen Dialog mit allen Bevölkerungsgruppen und Religionsgemeinschaften. Sie wollen sich für die Einrichtung von Lehrstühlen für islamische Theologie an deutschen Hochschulen, für die Ausbildung von deutschsprachigen Imamen und muslimischen Religionslehrern einsetzen. Nach der FDP sind die Muslime Teil der deutschen Gesellschaft und islamischer Religionsunterricht muss in deutscher Sprache an den Schulen erteilt werden.

Das Wahlprogramm der Linkspartei bestimmt zuerst den Zustand der Migranten: Die rassistische Diskriminierung und die Politik der sozialen Ausgrenzung sowie des sozialen und ethnischen selektiven Bildungssystems haben dazu geführt, dass Armut besonders unter Migrantinnen und Migranten zugenommen und sich verfestigt hat. Migrantinnen und Migranten sind fast doppelt so häufig von Erwerbslosigkeit betroffen, ihr Armutsrisiko ist dreimal höher als das der übrigen Bevölkerung. Diese Situation liegt an der unsozialen und ausgrenzenden Politik der bisherigen Bundesregierungen. Die Linkspartei will eine weltoffene Gesellschaft, ein respektvolles gesellschaftliches Miteinander in Anerkennung der Verschiedenheit aller Menschen und fordert gleiche Rechte auf soziale und politische Teilhabe für alle in Deutschland lebenden Menschen. Eine Migrations- und Integrationspolitik wird von der Linkspartei abgelehnt und die Quoten, Kontingente und Punktesysteme werden als Instrumente einer neokolonialen, selektiven Einwanderungspolitik angesehen. Deutschland braucht keine Verschärfungen des Aufenthaltsrechts und rassistische Debatten über angebliche Integrationsverweigerer. Alle hier geborenen Kinder und Jugendlichen sollen die deutsche Staatsbürgerschaft haben, ohne die Staatsbürgerschaft der Eltern ablehnen zu müssen. Die Linkspartei will die strukturellen Nachteile und Barrieren beim Zugang zu Bildung und Ausbildung, zum Arbeitsmarkt und zu sozialen Dienstleistungen beseitigen. Darüber hinaus fordert die Linkspartei die Erleichterung der Einbürgerungsmöglichkeiten und Mehrfachstaatsbürgerschaften, die Abschaffung des Deutsch-Tests beim Ehegattennachzug und im Aufenthaltsrecht, die Verbesserung und Ausweitung eines auf Freiwilligkeit basierenden Integrations- und Sprachkursangebots, die Anerkennung der im Ausland erworbenen Qualifikationen und die offenen Grenzen für alle Menschen. Der Familiennachzug von Kindern und Ehegatten darf nicht behindert werden. Die Linke betont besonders die Situation der Türken: Die europarechtswidrige Diskriminierung türkischer Staatsangehöriger muss beendet und ihre Rechte aus dem EU-Türkei-Assoziationsabkommen umfassend gewahrt werden. 
Die Grünen wollen ihre Integrationspolitik durch eine Politik der Inklusion vervollständigen. Die Kinder, Enkel und Urenkel der ersten Einwanderer-Generation müssen nicht ,integriert“ werden, weil sie nicht außerhalb in der deutschen Gesellschaft stehen und sie von Anfang an dazu gehören. Laut den Grünen kann Integration nicht von oben verordnet werden, sondern werden Integrations- und Inklusionspolitik gemeinsam mit der Zivilgesellschaft durch die Selbstbestimmung und Gleichberechtigung entwickelt. Die Grünen betonen die echte Chancengerechtigkeit bei Bildung und Arbeit und wollen die Qualität von Integrationskursen und Sprachförderung in Kitas verbessern, Deutschkurse im Inland für alle Interessierten öffnen, Mehrsprachigkeit als Ressource nutzen und ausländische Berufsabschlüsse leichter anerkennen. Sie fordern die schnelle und leichte Erlangung der deutschen Staatsbürgerschaft, ein erleichtertes Einbürgerungsverfahren für die erste Generation und die Abschaffung des Optionsmodells. Das Ziel der Grünen ist es, die eingebürgerten Menschen erneut ihre alte Staatsbürgerschaft zusätzlich anzunehmen, den Nachzug von ausländischen Ehegatten wieder zu erleichtern, den Sprachtest im Ausland als Vorbedingung abzuschaffen, das Aufenthaltsrecht $\mathrm{zu}$ modernisieren und die Einwanderung von Arbeitskräften grundsätzlich zu erleichtern. Alle einwandernden Arbeitskräfte sollen ihren Aufenthalt einfacher verlängern und verfestigen können. Den Familiennachzug im Aufenthaltsgesetz wollen die Grünen dem geltenden EU-Recht anpassen. Nach den Grünen haben Türkische Staatsangehörige und ihre Familienangehörigen, die in Deutschland leben, aufgrund des Assoziationsabkommens zwischen der Türkei und der heutigen EU Rechte, die denen von UnionsbürgerInnen nahekommen. Diesen Status wollen sie auch ausdrücklich gesetzlich verankern, z. B. im Aufenthalts-, Beschäftigungserlaubnis und Beamtenrecht.

\section{Rechtsextremismus}

Wegen der Bedrohung von Fremdenfeindlichkeit und Rechtsextremismus für die Demokratie wird dieses Problem von allen Wahlprogrammen der Parteien mehr oder weniger thematisiert. Andererseits stellt Rechtsextremismus, besonders im Zusammenhang mit den NSU-Morden, für ethnische Minderheiten eine große psychische Belastung dar. In der Gewichtung der Bekämpfung von Rechtsextremismus ergeben sich parteiliche Unterschiede. Dieses Problem wird von den Unionsparteien $(0,45 \%)$ und der FDP $(0,04)$ in ihren Wahlprogrammen sehr gering und oberflächlich bearbeitet. Dagegen wird dieses Thema von der Linkspartei $(1,83 \%)$, der SPD $(1,09 \%)$ und den Grünen (0,90\%) häufiger als bei der CDU-CSU und der FDP thematisiert (vgl. Tabelle 3). 
Tabelle 3: Rechtsextremismus in den Wahlprogrammen der Parteien

\begin{tabular}{|c|c|c|}
\hline Parteien & \multicolumn{2}{|c|}{ Rechtsextremismus } \\
\hline CDU-CSU & \% & Wörter \\
\hline SPD & 0,45 & $(192)$ \\
\hline FDP & 1,09 & $(477)$ \\
\hline DIE LINKE & 0,04 & $(17)$ \\
\hline DIE GRÜNEN & 1,83 & $(561)$ \\
\hline
\end{tabular}

Die CDU-CSU und die FDP sprechen vor allem von „Extremismus und Terrorismus“. Die Unionsparteien wollen mit dem Zusammenwirken von Verfassungsschutz und Polizei im Gemeinsamen Extremismus und Terrorismusabwehrzentrum die Schlagkraft der Sicherheitsbehörden stärken und sehen die Anti-Terror-Datei und die Rechtsextremismus-Datei im Kampf gegen den internationalen Terrorismus und gegen Rechtsextremismus als unverzichtbare Mittel für den schnellen Informationsaustausch zwischen den Sicherheits- und Verfassungsschutzbehörden an. Sie sprechen gleichzeitig vom Links- und Ausländerextremismus und setzen sich dafür ein, dass gegen jede Form von extremistischen und terroristischen Bestrebungen alle verfassungsund vereinsrechtlichen Mittel, wie etwa Vereinsverbote, konsequent genutzt werden.

Die FDP will eine wehrhafte Demokratie, die gegen alle extremistischen Bedrohungen gewappnet ist. Terrorismus und Extremismus sind weiterhin konsequent zu bekämpfen und zu verfolgen. Deshalb unterstützt sie den Ausbau und eine stärkere Koordinierung von Deradikalisierungsprogrammen. Extremismus darf nicht toleriert oder entschuldigt werden - egal ob er links, rechts oder religiös motiviert ist. Unfreiheit, Armut und Chancenlosigkeit werden von der FDP als die Gründe für politischen und religiösen Extremismus bezeichnet. Die Erinnerung an die beiden deutschen Diktaturen, an ihre Opfer, ihre Täter und den Widerstand gegen sie wird von den Liberalen als eine gesamtgesellschaftliche Aufgabe angesehen.

Die SPD Thematisiert das Thema „Rechtsextremismus“ unter dem Titel „Rechtsextremismus bekämpfen“. Sie sieht die von der rechtsextremistischen Terrorgruppe „NSU” begangenen zehn Morde an türkisch- bzw. griechischstämmigen Bürgern und die Sprengstoffanschläge als feige Angriff auf rechtschaffende Mitbürger und zugleich als ein Angriff auf die Demokratie. Nach den Sozialdemokraten haben die Arbeit der Untersuchungsausschüsse im Bund und in mehreren Ländern eine Vielzahl an Fehlern auf allen Ebenen offenbart und darum konnte die Terrorzelle nicht frühzeitig gestoppt werden. 
Die SPD fordert in diesem Sinne, das Vertrauen in die Sicherheitsbehörden dringend wiederherzustellen und will das erreichen durch rückhaltlose Aufklärung der Vorgänge, durch institutionelle Reformen und dadurch, dass wir jede Form von Rechtsextremismus frühzeitig und umfassend bekämpfen. Sie verspricht, dass die mit großem Engagement durch den NSUUntersuchungsausschuss des Bundestages entwickelten Folgerungen und Vorschläge in der nächsten Wahlperiode wirkungsvoll umgesetzt werden. Die SPD will auch den organisierten Rechtsextremismus bekämpfen, deshalb die NPD verbieten. Die NPD ist laut der Sozialdemokraten in aggressiver Form rassistisch, antisemitisch und fremdenfeindlich und missbraucht das Parteienprivileg der Verfassung. Die SPD will vor allem die Zivilgesellschaft durch Bildung, Ausbildung, Antidiskriminierungsmaßnahmen, Aufklärung und Schulung stärken und mit einem integrierten Konzept gegen Rechtsextremismus, Rassismus und Antisemitismus die Kräfte der Zivilgesellschaft, der Ermittlungsbehörden und der Politik in Kommunen, Land und Bund bündeln. Für die SPD ist der Kampf gegen Rechtsextremismus, Rassismus und Antisemitismus eine gesamtgesellschaftliche Daueraufgabe. Zudem will die SPD beim Kampf gegen Rechtsextremismus, Rassismus und Antisemitismus notwendige Anschlussförderungen sichern und gemeinsam mit der Zivilgesellschaft im Rahmen eines abgestimmten Aktionsplans ein neues Förderkonzept erarbeiten und umsetzen.

Die Linkspartei bezeichnet Rassismus, Nationalismus, Antisemitismus und eine allgemeine Ideologie der Ungleichheit der Menschen als das Kennzeichen der extremen Rechten. Nach den Linken wurzeln Rassismus und Antisemitismus tief in der Mitte der Gesellschaft. Die Linkspartei glauben daran, dass muslimische Menschen seit dem 11. September unter dem Generalverdacht terroristischer Umtriebe stehen und diese Situation zu struktureller Islamfeindlichkeit führt. Darum will die Linke den Dialog mit muslimischen und jüdischen Gemeinden und Vereinen suchen und sich gemeinsam gegen antimuslimischen Rassismus und Antisemitismus stellen. Die Linkspartei fordert eine systematische Erfassung der Straftaten mit rassistischem, antisemitischem oder islamfeindlichem Hintergrund und steht gegen Ungleichheitsideologien, jede Form von Rassismus, Antisemitismus, Islamfeindlichkeit, Demokratiefeindlichkeit und Neofaschismus ein. Die Bundesprogramme gegen rechts müssen dauerhaft und umfassend gefördert und alle Hindernisse für die Programme beseitigt werden. Die Linke setzt sich darüber hinaus für eine antifaschistische Erinnerungskultur ein und fordert ein Verbot der NPD und aller weiteren neofaschistischen Organisationen. Nach der Angesichts der Linkspartei kann das Problem von Rechtsextremismus und Rassismus allein nicht gelöst werden. Man muss staatliche Unterstützung für die Strukturen der Extremen Rechten verhindern.

Im Wahlprogramm von Bündnis 90/Die Grünen wird Rechtsextremismus mit dem Slogan „Entschlossen gegen Rechtsextremismus, 
Rassismus und gruppenbezogene Menschenfeindlichkeit vorgehen" bearbeitet. Sie behaupten, dass Alte und neue Nazis vielerorts bereits tief verankert sind, dass sie durch ihr Auftreten vielerorts „Angst-Räume“ geschaffen haben und dass gruppenbezogene Menschenfeindlichkeit, wie Rassismus, Antisemitismus, Antiziganismus, antimuslimischer Rassismus usw. nicht nur am rechten Rand, sondern auch in der Mitte der Gesellschaft anzutreffen sind. Die Grünen versprechen, staatlich zu unterstützen, wo immer BürgerInnen sich gegen Nazis durch Bildungs- und Beratungsarbeit, durch Demos und friedliche Blockaden von Nazi-Aufmärschen engagieren. Sie wollen für die Demokratieförderung und den Kampf gegen Rechtsextremismus und Menschenfeindlichkeit dauerhaft jährlich 50 Mio. Euro bereitstellen und zusammen mit den Ländern und der Zivilgesellschaft eine neue Förderstruktur entwickeln und eine langfristige Planungsperspektive erhalten. Die Grünen betonen, dass der Staat Rechtsextremismus und Rassismus mit allen rechtsstaatlichen Mitteln bekämpfen muss, dass Rassismus und andere Formen von gruppenbezogener Menschenfeindlichkeit in Deutschland keinen Platz haben und dass die NPD eine verfassungsfeindliche, menschenverachtende und rechtsextreme Partei ist. Darum fordern die Grünen ein Verbot der NPD und wollen die Grauzonen zwischen rechtskonservativer und rechtsextremer Ideologie ausleuchten.

\section{Diskriminierung}

Es handelt sich bei der Diskriminierung von Migranten um die unterschiedlichen Formen, in denen sich die Diskriminierung in vielen gesellschaftlichen Bereichen wahrnimmt. In diesem Sinne werden Migranten meistens mehrfach diskriminiert. Das heißt, „dass Benachteiligungen häufig nicht nur aufgrund der ethnischen Herkunft erfolgen, sondern dass zusätzlich andere Merkmale wie etwa Geschlecht und Religion zum Tragen kommen. Dies trifft insbesondere zu auf muslimische Migrantinnen, die ein Kopftuch tragen. Diese werden in vielen Bereichen des täglichen Lebens stark ausgegrenzt" (Schlaab, 2010: 28).

Obwohl die Diskriminierung in der deutschen Gesellschaft eine Realität ist, wird dieses Thema meistens nur von den linken Parteien thematisiert. Im Wahlprogramm der Unionsparteien wird das Wort „Diskriminierung“ nur zwei Mal verwendet und wird von der Diskriminierung der Menschen auf Grund ihres Alters und von der Diskriminierung anderer Formen der Partnerschaft gesprochen. Im Regierungsprogramm der Unionsparteien ist es keine Rede von der Form der Diskriminierung gegen die Migranten. Dagegen impliziert das Wahlprogramm der FDP nur mit 0,04\% dieses Thema. Die Liberalen wenden sich gegen jegliche Diskriminierung aufgrund von Religion, ethnischer Herkunft, Geschlecht, Behinderung, Alter oder sexueller Orientierung. Sie wollen in Zukunft besonders die Menschen unterstützen, die von Ausgrenzung, Diskriminierung und Gewalt betroffen sind. 
Tabelle 4: Diskriminierung in den Wahlprogrammen der Parteien

\begin{tabular}{|c|c|c|}
\hline Parteien & \multicolumn{2}{|c|}{ Diskriminierung } \\
\hline CDU-CSU & $\%$ & Wörter \\
\hline SPD & - & $(278)$ \\
\hline FDP & 0,63 & $(37)$ \\
\hline DIE LINKE & 0,04 & $(91)$ \\
\hline DIE GRÜNEN & 0,29 & $(787)$ \\
\hline
\end{tabular}

Im Wahlprogramm der SPD liegt das Diskriminierungsproblem bei 0,63\%. Die Sozialdemokraten streben eine Gesellschaft an, in der jeder Mensch sich frei entfalten kann, ohne die Würde und Freiheit anderer zu verletzen. Sie betonen, dass sie sich jeder Form der Diskriminierung widersetzen. Jeder Form des Sexismus und allen Formen von frauenverachtendem Verhalten und Gewalt gegen Frauen sagen sie den Kampf an. Die SPD will eine Gesellschaft, die sich jeder Form der Diskriminierung widersetzt und eine Kultur des Widerspruchs fördert, wenn bewusst oder unbewusst Rechte und Würde des Menschen verletzt werden und einen Staat, der diese Würde und Rechte wirksam schützt. Die SPD verspricht, jede Form der Diskriminierung, ob wegen Herkunft, Geschlecht, sexueller Identität, Religion und Weltanschauung, Behinderung oder Alter zu bekämpfen, das Allgemeine Gleichbehandlungsgesetz (AGG) weiterzuentwickeln und eine bessere Finanzierung für die Antidiskriminierungsstelle des Bundes sicherzustellen. Gleichzeitig werden im Wahlprogramm der SPD die Achtung und Schätzung der Religionsfreiheit und die religiöse und weltanschauliche Vielfalt in Deutschland betont.

Im Wahlprogramm 2013 der Linkspartei wird die Diskriminierung noch kürzer $(0,29 \%)$ als im ihren früheren Wahlprogrammen behandelt (vgl. Acar, 2010: 112) und werden die soziale Ausgrenzung und ökonomische Armut als die Ergebnisse der Diskriminierung unterstrichen. Nach den Linken hängt die Diskriminierung vom alltäglichen Rassismus ab. Wegen der Politik bisheriger Regierungen geht es laut der Linken um die rassistische Diskriminierung, Politik und das rassistische Bildungssystem. Die Linkspartei hatte in ihren Wahlprogrammen 2005 und 2009 das Problem Diskriminierung noch umfangreicher diskutiert und die Notwendigkeit eines Antidiskriminierungsgesetzes (2005) und die Diskriminierung gegen Türken und Kurden (2009) zur Sprache gebracht (vgl. Acar, 2010: 213). Die Linke hebt auch im Wahlprogramm 2013 die europarechtswidrige Diskriminierung türkischer Staatsangehöriger hervor.

Die Grünen thematisieren die Diskriminierung in ihrem Wahlprogramm ziemlich lang $(0,87 \%)$. Sie streben, eine offene Gesellschaft zu schaffen, in der 
jede und jeder ohne Diskriminierung, ohne Angst vor Unterdrückung - sei es durch den Staat, durch menschenrechts- und demokratiefeindliche Bewegungen, durch private Einrichtungen, durch die eigene Familie - leben, arbeiten und handeln kann. Die Grünen versprechen, sich mit mit aller Kraft gegen jegliche Diskriminierung $\mathrm{zu}$ wenden und das anonymisierte Bewerbungsverfahren im öffentlichen Dienst zu verbreiten. Menschen können sich ungehindert einmischen - ohne dabei Angst vor Nachteilen oder Diskriminierung haben zu müssen. Sie akzeptieren nicht, dass Menschen weiter von demokratischen Rechten ausgeschlossen bleiben, obwohl sie schon lange hier leben. Nach den Grünen bringt Diskriminierung keinen Sicherheitsgewinn und Ausweiskontrollen und Maßnahmen von Sicherheitsbehörden gegen die Menschen ohne deutschen Pass oder mit einer dunkleren Hautfarbe sind diskriminierend und nach Verfassungs- und Völkerrecht verboten. Sie wollen dieses Verbot von diskriminierendem Ethnic Profiling klar gesetzlich regeln und Diversität und interkulturelle Öffnung von Verwaltung und Justiz gezielt fördern. Polizeibeamte müssen zudem verstärkt Antidiskriminierungsschulungen erhalten und die Polizei muss sich stärker interkulturell öffnen. Staat und Zivilgesellschaft müssen sich gegen alle Formen von Diskriminierung wenden. Ein wichtiges Ziel inklusiver Politik ist laut der Grünen die religiöse und weltanschauliche Gleichberechtigung aller Menschen.

\section{E. EU-Beitrittsfrage der Türkei}

Sowohl Türken als auch türkischstämmige Deutschen reagieren auf politische Aussagen und Themen viel emotionaler als die deutsche Bevölkerung. Zu diesen Themen zählt der EU-Beitritt der Türkei. Die Frage des EU-Beitritts der Türkei ist in Deutschland ein zentrales und spannendes außenpolitisches Thema, das sehr emotional diskutiert wird. Besonders in Zeiten vor Wahlen wird die Haltung der Parteien zum EU-Beitritt der Türkei von den türkischen Selbstorganisationen immer wieder thematisiert.

Tabelle 5: EU-Beitritt der Türkei in den Wahlprogrammen der Parteien

\begin{tabular}{|c|c|c|}
\hline Parteien & \multicolumn{2}{|c|}{ EU-Beitrittsfrage der Türkei } \\
\hline & $\%$ & Wörter \\
\hline CDU-CSU & 0,27 & $(116)$ \\
\hline SPD & 0,09 & $(43)$ \\
\hline FDP & 0,18 & $(70)$ \\
\hline DIE LINKE & - & - \\
\hline DIE GRÜNEN & 0,32 & $(293)$ \\
\hline
\end{tabular}


Diese Frage wird im Wahlprogramm der CDU-CSU unter dem Slogan „Beziehungen der Türkei auf eine neue Ebene heben“ diskutiert. Die Unionsparteien lehnen unbedingt eine EU-Mitgliedschaft der Türkei ab, obwohl die strategische und wirtschaftliche Bedeutung der Türkei für Europa und die vielfältigen Beziehungen zwischen den Menschen in beiden Ländern betont werden. Als Gründe für die Ablehnung werden genannt, dass die Türkei die Voraussetzungen für einen EU-Beitritt nicht erfüllt und angesichts der Größe des Landes und seiner Wirtschaftsstruktur zudem die Europäische Union überfordert wäre. Sie wollen daher die Beziehungen zwischen der Europäischen Union und der Türkei weiter vertiefen und eine möglichst starke Kooperation zwischen der Europäischen Union und der Türkei sowie eine enge strategische Zusammenarbeit in außen- und sicherheitspolitischen Fragen. Die Unionsparteien verzichten auch in ihrem Wahlprogramm 2013 auch auf die privilegierte Partnerschaft, die ,als eine Alternative zur Vollmitgliedschaft der Türkei von Angela Merkel und Wolfgang Schäuble seit 2004 vorgeschlagen und im Wahlprogramm 2005 erklärt wurde“ (Acar, 2010: 117). Auf diese Haltung der Unionsparteien reagiert die türkische Bevölkerung in Deutschland, weil die Abweisung der Türkei sehr persönlich wahrgenommen wird. „Dieses Gefühl der Ablehnung wird auf die eigene Person übertragen: Wer gegen die EU-Mitgliedschaft der Türkei ist, ist auch gegen die Türken in Deutschland“ (Wilamowitz-Moellendorff 2005: 44).

Dagegen hat die SPD gegenüber der EU-Mitgliedschaft der Türkei eine klare Haltung. Sie vertritt die Meinung, dass Europa zudem weiterhin seine Türen für neue Mitglieder offen halten muss, weil sich die Erweiterungspolitik der EU als vorausschauende Friedenspolitik bewährt hat. Sie tritt dafür ein, dass die EU die Verhandlungen mit der Türkei mit dem klaren Ziel eines EUBeitritts der Türkei weiterführt und ihnen neue Dynamik verleiht. Ein EUBeitritt der Türkei ist laut der SPD im wechselseitigen politischen und ökonomischen Interesse und kann wichtige kulturelle Brücken schlagen. Es ist die Erwartung der SPD, dass Europa zu seinem Wort gegenüber den Ländern stehen muss, die bereits Beitrittsverhandlungen mit der EU führen oder eine Beitrittsperspektive erhalten haben.

Die FDP unterstricht im Zusammenhang der EU-Mitgliedschaft der Türkei, dass Deutschland mit der Türkei seit Jahrzehnten eine tiefgreifende Partnerschaft und die gemeinsame Mitgliedschaft in der NATO verbindet und dass die EU und die Türkei seit 2005 ergebnisoffen über eine türkische EUMitgliedschaft verhandeln. Es wird erklärt, dass die FDP an der Beitrittsperspektive für die Türkei festhält und dass es aber jedoch - wie in allen Fällen - keine Rabatte bei der Erfüllung der Beitrittskriterien geben darf. In diesem Sinne wollen die Liberalen die Türkei ermutigen, den notwendigen Reformprozess in Richtung Rechtsstaat und Menschenrechte entschlossener voranzutreiben. 
Die Linkspartei macht in ihren Wahlprogrammen $\mathrm{zu}$ den Bundestagswahlen 2002, 2005, 2009 und 2013 aus dem EU-Beitritt der Türkei kein Thema. Sie hat nur in ihrem Programm zu den Europawahlen 2004 erklärt, dass ,sie die Aufnahme von Verhandlungen über einen EU-Beitritt der Türkei befürwortet, wenn diese die Kopenhagener Kriterien erfüllt und vor allem die Menschenrechtssituation verbessert" (Acar, 2010: 119)

Die Grünen haben eine positive Haltung zum EU-Beitritt der Türkei. Sie vertreten im Allgemeinen die Meinung, dass die Europäische Union, 50 Jahre nach dem entsprechenden Assoziationsabkommen, endlich für neuen Schwung in den Beitrittsverhandlungen mit der Türkei sorgen muss und diese fair, ergebnisorientiert und auf Augenhöhe geführt werden müssen. Für die Grünen stehen hierbei Demokratie und Menschenrechte vor Ort im Vordergrund. Es wird von den Grünen festgestellt, dass es in der Türkei nach wie vor Defizite, z. B. im Fall von Pressefreiheit, Frauenrechten und Minderheitenschutz gibt und dass gerade ein ernsthafter Beitrittsprozess jedoch den meisten Reformerfolg verspricht. Die Grünen wollen die Zugehörigkeit zur EU nicht von religiösen Identitäten abhängig machen. Es wird erklärt, dass es bei den bestehenden Kriterien keine Abstriche geben darf und das Ziel der Verhandlungen der Beitritt ist, sobald diese Kriterien erfüllt sind. Die Grünen wollen andererseits, dass die Visumspflicht für Menschen aus den Ländern der Östlichen Partnerschaft, Russland, Kosovo und der Türkei zügig aufgehoben wird.

\section{F. Die Parteien im Vergleich}

Die Rolle der Parteienangebote an die Deutschen türkischer Herkunft haben besondere Prägekraft für ihr Wahlverhalten, weil dieses durch politische Faktoren, vor allem durch die Strategien der Parteien, für ihre Interessenlage bestimmt wird. Die Wählerschaft will vor allem wissen, welche Partei ihre Interessen vertritt. „Was die Wähler durch ihre Präferenz für bestimmte politische Parteien als materielles Interesse äußern, ist ihr Interesse“ (Elff, 2006: 55). In diesem Zusammenhang haben die Wahlprogramme der Parteien große Bedeutungen, weil die Wahlprogramme der Parteien den türkischstämmigen Wählern die Möglichkeit bieten, sich über die Zielsetzungen der verschiedenen Parteien zu informieren und die verschiedenen Positionen mit ihren eigenen Interessen $\mathrm{zu}$ vergleichen. Die gegensätzlichen Haltungen der Parteien im Politikfeld Migration und Integration zeigen sich auch in ihren Programmen für die Bundestagswahl 2013.

Die ideologischen Grundpositionen der Wahlprogramme der Parteien 2013 in der Migrations- und Integrationspolitik sind sehr deutlich. Die Unionsparteien sind immer sehr kritisch, wenn es um die Zuwanderung und Integration geht. Die SPD, Grünen und Linkspartei sind dagegen ausländerfreundlich und positiv. Die Position der FDP ist liberal, aber hängt von ihrer Koalitionsabsicht mit den Unionsparteien ab. 
Die wahlprogrammatischen Positionen der Parteien zeigen, dass die Linksparteien die Interessen ethnischer oder kultureller Minderheiten vertreten, während die Rechtsparteien eine negative Gegenpositionen oder sozialkonservative Positionen vertreten. Grüne, Linkspartei und SPD profilieren sich als Parteien, die der offene Gesellschaft, dem Kampf gegen Rechtsextremismus und Diskriminierung und der Türkei sehr positiv gegenüber stehen, gefolgt von der FDP, während die Unionsparteien gegenüber diesen Themen eindeutig die negative Gegenposition haben. Besonders in den Themenbereichen „Rechtsextremismus“, „Diskriminierung“ und „EU-Beitritt der Türkei“ werden die Parteien von den eingebürgerten Türken sowohl prospektiv als auch retrospektiv bewertet, d.h. die Beurteilung dieser Sachfragen beruht auf vergangenen Erfahrungen und auf zukünftigen Erwartungen. Die linken Parteien wirken durch ihre klare Haltung und ihre positive wahlprogrammatische Aussage $\mathrm{zu}$ diesen Themen immer positiv auf Türkischstämmige und sind für die Integration von Ausländern offener und toleranter.

Diese quantitative und qualitative Inhaltsanalyse zeigt auch, wie weit die Parteien programmatisch voneinander entfernt sind. Die größte programmatische Distanz ergibt sich zwischen den Unionsparteien, der Linkspartei und den Grünen, dagegen ergeben sich nur geringe Distanzen zwischen der Linkspartei, den Grünen und der SPD.

\section{RESÜMEE}

In der vorliegenden Untersuchung wurden die Wahlprogramme der im Bundestag vertretenden fünf Parteien (CDU-CSU, SPD, FDP, Die Linke und Bündnis 90/Die Grünen) zur Bundestagswahl 2013 hinsichtlich der Integrationspolitik, des Rechtsradikalismus, der Diskriminierung und der EUBeitrittsfrage der Türkei mit Hilfe der quantitativen und qualitativen Methoden inhaltlich analysiert. Es sollte deutlich geworden sein, mit welchen Positionen und Aussagen hinsichtlich der oben genannten Themen die Parteien in die Bundestagswahl am 22.09.2013 gehen. Die Länge der Wahlprogramme und der inhaltlichen Schwerpunkte unterscheiden sich. Die Linkspartei hat das kürzeste, Bündnis 90/Die Grünen das längste Wahlprogramm.

Die Inhaltsanalyse der Wahlprogramme von CDU-CSU zeigt, dass die Unionsparteien für die türkischstämmige Wählerschaft nicht mehr attraktiv und nicht mehr vielversprechend sind. Die CDU-CSU haben eine negative Haltung gegenüber dem kommunalen Wahlrecht, der Doppelstaatsbürgerschaft und der EU-Beitrittsfrage der Türkei. Die migrations- und integrationsspezifischen Probleme der Türken und türkischstämmigen Bürger sind weitere von den Unionsparteien nicht berücksichtigte politische Themen. Dagegen betonen sie die Werte der deutschen Gesellschaft und damit die Integration als eine Pflicht. Sie bringen auch den Kampf gegen Diskriminierung und Rechtsextremismus nicht zur Sprache. 
Dagegen verortet die SPD mit der progressiven Position im Gegenpol. Es wird deutlich, dass migrations- und integrationsspezifische Themen im Wahlprogramm der SPD umfangreicher als im Programm der Unionsparteien bearbeitet werden und dass politische Probleme, die von den Türken oder/und Deutsch-Türken besonders wahrgenommen werden, und ihre Lösungen klarer erklärt werden. Die soziale und politische Gleichberechtigung der Menschen und die Erleichterungen der Integrationspflichten in unterschiedlichen Bereichen bilden den Kern der Integrationspolitik. Mit der Annahme der Doppelstaatsbürgerschaft, des kommunales Wahlrechts und der EUMitgliedschaft der Türkei ist die SPD die bedeutendste Partei für die Türken. Die Haltung der SPD gegenüber dem Kampf gegen Rechtsextremismus und Diskriminierung verläuft parallel zu den sozialdemokratischen und menschenrechtlichen Werten.

Die FDP liegt bei der Integrationspolitik häufig näher bei den linken Parteien. Sie stellt fest, dass die Partizipationsmöglichkeiten mit der Annahme des kommunales Ausländerwahlrechts die Integration erleichtern. Die Zulassung der doppelten Staatsbürgerschaft, die Betonung der offenen und liberalen Gesellschaft, des offenen Dialog mit allen Minderheiten und Religionen und des islamischen Religionsunterrichts stehen im Mittelpunkt der Integrationspolitik der FDP. Obwohl Die Haltung der FDP gegenüber der Bekämpfung des Rechtsextremismus, der Diskriminierung und der EUMitgliedschaft der Türkei positiv ist, werden diese Themen wegen einer Koalitionsmöglichkeit mit der Unionsparteien oberflächlich thematisiert.

Das Wahlprogramm der Linkspartei unterscheidet sich deutlich von anderen Wahlprogrammen und tritt für die gleichen politischen, sozialen und kulturellen Rechte aller Menschen in Deutschland ein. Darum wird Integrationspolitik von den Linken abgelehnt. Die Linkspartei fordert kein kommunales Wahlrecht, sondern das Wahlrecht für die Migranten auf allen Ebenen. Sie fordert auch keine Doppelstaatsbürgerschaft, sondern die Mehrfachstaatsbürgerschaften. Das Programm befindet sich mit der Forderungen (der Erleichterung der Einbürgerungsmöglichkeiten, der Abschaffung der Deutsch-Tests, der offenen Grenzen für alle Menschen und dem Recht des Familiennachzuges von Kindern und Ehegatten) im linken Pol. Die Bekämpfung des Rechtsextremismus und der Diskriminierung wird von den Linken sehr detailliert bearbeitet. Die Linkspartei spricht auch vom antimuslimischen Rassismus, Neofaschismus und von der Islamfeindlichkeit.

In der Analyse des Wahlprogramms zeigt sich, dass Bündnis 90/Die Grünen klare und weitgehende Antworten zur besonderen Problemlage der Migranten haben. Wegen ihrer klaren Positionierung gegen Rechtsextremismus und Diskriminierung und ihrer positiven Haltung gegenüber der EUMitgliedschaft der Türkei sind die Grünen eine der bedeutendsten Parteien für die Wahlberechtigten türkischer Herkunft. Sie sprechen sich für den Doppelpass, das Ausländerwahlrecht, die Inklusionspolitik anstatt der 
Integrationspolitik, die Rechte von Türken aufgrund des Assoziationsabkommens zwischen der Türkei und EU und die Gleichstellung des Islams mit Christentum und Judentum aus.

Als Ergebnis ist festzustellen, dass die integrationspolitischen Themen und die Lösungen besonderer Probleme der Migranten von den linken Parteien in ihren Wahlprogrammen zur Bundestagswahl 2013 in einer erwartbaren Perspektive (besonders bei den Themen Rechtsextremismus, Diskriminierung, Wahlrecht und Zulassung der Doppelstaatsbürgerschaft) behandelt werden. Dagegen werden die ausgewählten Themen von den Unionsparteien auch erwartungsgemäß sehr konservativ formuliert.

\section{LITERATUR}

ACAR, Mustafa (2010), Das Wahlverhalten der türkischstämmigen Wahlberechtigten in der Bundesrepublik Deutschland: Eine Untersuchung zur Erklärung des politischen Verhaltens von Deutsch-Türken bei den Bundestagswahlen 2002 und 2005, Verlag Dr. Kovac, Hamburg.

ACAR, Mustafa (2012), Politische Einstellungen von türkischen Migranten in Hamburg: Eine empirische Untersuchung zum politischen Leben von Türken, Verlag Dr. Kovac, Hamburg.

ATTESLANDER, Peter (2003), Methoden der empirischen Sozialforschung, WDEG, Berlin.

ATTESLANDER, Peter (2010), Methoden der empirischen Sozialforschung, 13. neue bearbeitete und erweiterte Auflage, Erich Schmidt Verlag GmbH-Co., Berlin.

BERELSON, Bernard (1952), Content Analysis in Communication Research, Free Press, New York.

BORTZ, Jürgen und DÖRING, Nicola (2005), Forschungsmethoden und Evaluation für Human- und Sozialwissenschaftler, 3. überarbeitete Auflage, Springer Medizin Verlag, Heidelberg.

DEBUS, Marc (2007), „Die programmatische Entwicklung der deutschen Parteien auf Bundes- und Landesebene zwischen den Bundestagswahlen 1998 und 2005“, Die Bundestagswahl 2005: Analysen des Wahlkampfes und der Wahlergebnisse, (Hrsg.) Frank Brettschneider, Oskar Niedermayer, Bernhard Weßels, VS Verlag, Wiesbaden, s. 43-65.

DIEKMANN, Andreas (2001), Empirische Sozialforschung, 7.Aufl., Rowohlt, Reinbek bei Hamburg.

ELFF, Martin (2006), Politische Ideologien, soziale Konflikte und Wahlverhalten. Die Bedeutung politischer Angebote der Parteien für den Zusammenhang zwischen sozialen Merkmalen und Parteipräferenzen in zehn westeuropäischen Demokratien, Nomos Verlagsgesellschaft, Baden-Baden.

FLICK, Uwe (1991), Handbuch qualitativer Sozialforschung: Grundlagen, Konzepte, Methoden und Anwendungen, Psychologie-VerlagsUnion, München. 
FRÜH, Werner (2007), Inhaltsanalyse, UVK Verlagsgesellschaft, Konstanz.

GLÄSER, Jochen und LAUDEL, Grit (2006), Experteninterviews und qualitative Inhaltsanalyse, VS Verlag für Sozialwissenschaften, Wiesbaden.

KLINGEMANN, Hans-Dieter und VOLKENS, Andrea (2001), „Struktur und Entwicklung von Wahlprogrammen in der Bundesrepublik Deutschland 1949-1998“, Parteiendemokratie in Deutschland, (Hrsg.) W. Oscar Gabriel, Oskar Niedermayer, Richard Stöss, Westdeutscher Verlag, Bonn, Opladen, s. 507-527.

KLINGEMANN, Hans-Dieter (1989), „Die programmatischen Profile der politischen Parteien in der Bundesrepublik Deutschland. Eine quantitative Inhaltsanalyse der Wahlprogramme von SPD, FDP und CDU von 1949 bis 1987“, Konfliktpotentiale und Konsensstrategien. Beiträge zur politischen Soziologie der Bundesrepublik, (Hrsg.) Dietrich Herzog und Bernhard Wessels, Westdeutscher Verlag, Opladen, s. 99-115.

KROMREY, Helmut (1998), Empirische Sozialforschung, Leske + Budrich, Opladen.

LAMNEK, Siegfried (2005), Qualitative Sozialforschung, Beltz, Weinheim.

MAYRING, Philipp (2002), Einführung in die qualitative Sozialforschung, Beltz Verlag, Weinheim, Basel.

MAYRING, Philipp (1988), Qualitative Inhaltsanalyse, Grundlagen und Techniken, Beltz, Weinheim.

MAYRING, Philipp (1990), Qualitative Inhaltsanalyse. Grundlage und Techniken, Deutscher Studienverlag, Weinheim.

KORTE, Karl-Rudolf (2003), Wahlen in der Bundesrepublik Deutschland, 4. überarbeitete und aktualisierte Auflage, Bonifatius Druck Buch Verlag, Paderborn.

PAPPI, Franz Urban und SHIKANO, Susumu. (2004), Ideologische Signale in den Wahlprogrammen der deutschen Bundestagsparteien 1980 bis 2002, Arbeitspapiere Mannheimer Zentrum für Europäische Sozialforschung, Nr. 76, ISSN 1437-8574, Mannheim.

SCHLAAB, Markus (2010), „Realität der Diskriminierung in Deutschland: Vermutungen und Fakten", Rassismus und Diskriminierung in Deutschland, (Hrsg.) Heinrich-Böll-Stiftung, Dossier, Berlin, s. 27-32.

STAMMEN, Theo (1986), Programme der politischen Parteien in der Bundesrepublik Deutschland, 4. Auflage, Bayerische Landeszentrale für Politische Bildungsarbeit, München.

STEINKE, Ines (1999), Kriterien qualitativer Forschung: Ansätze zur Bewertung qualitativempirischer Sozialforschung, Juventa, Weinheim.

VOGEL, Dita und WÜST, Andreas (2003), „Paradigmenwechsel ohne Instrumentenwechsel? Kontinuität und Wandel im Politikfeld Migration“, 
Sozial- und Wirtschaftspolitik unter Rot-Grün, (Hrsg.) Antonia Gohr und Martin Seeleib-Kaiser, Westdeutscher Verlag, Wiesbaden, s. 265-286.

VOLKENS, Andrea (2002), Handbuch zur Inhaltsanalyse programmatischer Dokumente von Parteien und Regierungen in der Bundesrepublik Deutschland, FS III 02-203, Verlag Wissenschaftszentrum, Berlin.

VOLKENS, Andrea (1989), „Parteiprogrammatik und Einstellungen politischer Eliten: Konsens- und Konfliktstrukturen in Wahlprogrammen“, Konfliktpotentiale und Konsensstrategien. Beiträge zur politischen Soziologie der Bundesrepublik, (Hrsg.) Dietrich Herzog und Bernhard Wessels, Westdeutscher Verlag, Wiesbaden, s. 116-144.

WILAMOWITZ-MOELLENDORFF, Ulrich (2005), „Die Rolle der türkischen Minderheit in Deutschland“, Schriften zur Europäischen Integration, Europa und die Türkei: Privilegierte Partnerschaft statt EU-Mitgliedschaft, http://www.cducsu.Eu/images/stories/docs/tuerkei 260805.pdf, abgerufen: 23.06.2013. 